\title{
The Best Constant of Sobolev Inequality Corresponding to Clamped Boundary Value Problem
}

\section{Kohtaro Watanabe, ${ }^{1}$ Yoshinori Kametaka, ${ }^{2}$ Hiroyuki Yamagishi, ${ }^{3}$ Atsushi Nagai, ${ }^{4}$ and Kazuo Takemura ${ }^{4}$}

${ }^{1}$ Department of Computer Science, National Defense Academy, 1-10-20 Hashirimizu, Yokosuka 239-8686, Japan

${ }^{2}$ Division of Mathematical Sciences, Graduate School of Engineering Science, Osaka University, 1-3 Machikaneyama-cho, Toyonaka 560-8531, Japan

${ }^{3}$ Tokyo Metropolitan College of Industrial Technology, 1-10-40 Higashi-ooi, Shinagawa, Tokyo 140-0011, Japan

${ }^{4}$ Department of Liberal Arts and Basic Sciences, College of Industrial Technology, Nihon University, 2-11-1 Shinei, Narashino 275-8576, Japan

Correspondence should be addressed to Kohtaro Watanabe, wata@nda.ac.jp

Received 14 August 2010; Accepted 10 February 2011

Academic Editor: Irena Rachůnková

Copyright (C) 2011 Kohtaro Watanabe et al. This is an open access article distributed under the Creative Commons Attribution License, which permits unrestricted use, distribution, and reproduction in any medium, provided the original work is properly cited.

Green's function $G(x, y)$ of the clamped boundary value problem for the differential operator $(-1)^{M}(d / d x)^{2 M}$ on the interval $(-s, s)$ is obtained. The best constant of corresponding Sobolev inequality is given by $\max _{|y| \leq s} G(y, y)$. In addition, it is shown that a reverse of the Sobolev best constant is the one which appears in the generalized Lyapunov inequality by Das and Vatsala (1975).

\section{Introduction}

For $M=1,2,3, \ldots, s>0$, let $H\left(=H_{0}^{M}(-s, s)\right)$ be a Sobolev (Hilbert) space associated with the inner product $(\cdot, \cdot)_{M}$ :

$$
\begin{gathered}
H=H(M)=\left\{u \mid u^{(M)} \in L^{2}(-s, s), u^{(i)}( \pm s)=0(0 \leq i \leq M-1)\right\}, \\
(u, v)_{M}=\int_{-s}^{s} u^{(M)}(x) \bar{v}^{(M)}(x) d x, \quad\|u\|_{M}^{2}=(u, u)_{M} .
\end{gathered}
$$


The fact that $(\cdot, \cdot)_{M}$ induces the equivalent norm to the standard norm of the Sobolev (Hilbert) space of $M$ th order follows from Poincaré inequality. Let us introduce the functional $S(u)$ as follows:

$$
S(u)=\frac{\left(\sup _{|y| \leq s}|u(y)|\right)^{2}}{\|u\|_{M}^{2}} .
$$

To obtain the supremum of $S$ (i.e., the best constant of Sobolev inequality), we consider the following clamped boundary value problem:

$$
\begin{gathered}
(-1)^{M} u^{(2 M)}=f(x) \quad(-s<x<s) \\
u^{(i)}( \pm s)=0 \quad(0 \leq i \leq M-1) .
\end{gathered}
$$

Concerning the uniqueness and existence of the solution to $(\operatorname{BVP}(M))$, we have the following proposition. The result is expressed by the monomial $K_{j}(x)$ :

$$
K_{j}(x)=K_{j}(M ; x)= \begin{cases}\frac{x^{2 M-1-j}}{(2 M-1-j) !} & (0 \leq j \leq 2 M-1), \\ 0 & (2 M \leq j) .\end{cases}
$$

Proposition 1.1. For any bounded continuous function $f(x)$ on an interval $-s<x<s,(\operatorname{BVP}(M))$ has a unique classical solution $u(x)$ expressed by

$$
u(x)=\int_{-s}^{s} G(x, y) f(y) d y \quad(-s<x<s)
$$

where Green's function $G(x, y)=G(M ; x, y)(-s<x, y<s)$ is given by

$$
\begin{aligned}
& G(x, y) \\
& =\frac{(-1)^{M}}{2}\left[K_{0}(|x-y|)+D^{-1}\left\{\left|\begin{array}{c|c|}
K_{i+j}(2 s) & K_{i}(s-y) \\
\hline K_{j}(s+x) & 0
\end{array}\right|+\left|\begin{array}{c|c}
K_{i+j}(2 s) & K_{i}(s+y) \\
\hline K_{j}(s-x) & 0
\end{array}\right|\right\}\right] \\
& =(-1)^{M} D^{-1} \mid \begin{array}{c|c}
K_{i+j}(2 s) & K_{i}(s+x \wedge y) \\
\hline K_{j}(s-x \vee y) & 0
\end{array} \quad(-s<x, y<s) .
\end{aligned}
$$

$D$ is the determinant of $M \times M$ matrix $\left(K_{i+j}\right)(2 s)(0 \leq i, j \leq M-1), x \wedge y=\min (x, y)$, and $x \vee y=\max (x, y)$. 
With the aid of Proposition 1.1, we obtain the following theorem. The proof of Proposition 1.1 is shown in Appendices A and B.

Theorem 1.2. (i) The supremum $C(M ;-S, s)$ (abbreviated as $C(M)$ if there is no confusion) of the Sobolev functional $S$ is given by

$$
C(M ;-s, s)=\sup _{u \in H, u \neq 0} S(u)=\max _{|y| \leq s} G(y, y)=G(0,0)=\frac{s^{2 M-1}}{2^{2 M-1}(2 M-1)\{(M-1) !\}^{2}} .
$$

Concretely,

$$
C(1,-s, s)=\frac{s}{2}, C(2,-s, s)=\frac{s^{3}}{24}, C(3,-s, s)=\frac{s^{5}}{640}, C(4,-s, s)=\frac{s^{7}}{32256}, \ldots
$$

(ii) $C(M ;-s, s)$ is attained by $u=G(x, 0)$, that is, $S(G(x, 0))=C(M ;-s, s)$.

Clearly, Theorem 1.2(i), (ii) is rewritten equivalently as follows.

Corollary 1.3. Let $u \in H$, then the best constant of Sobolev inequality (corresponding to the embedding of $H$ into $L^{\infty}(-s, s)$ )

$$
\left(\sup _{|y| \leq s}|u(y)|\right)^{2} \leq C \int_{-s}^{s}\left|u^{(M)}(x)\right|^{2} d x
$$

is $C(M ;-s, s)$. Moreover the best constant $C(M ;-s, s)$ is attained by $u(x)=c G(x, 0)$, where $c$ is an arbitrary complex number.

Next, we introduce a connection between the best constant of Sobolev- and Lyapunovtype inequalities. Let us consider the second-order differential equation

$$
u^{\prime \prime}+p(x) u=0 \quad(-s \leq x \leq s)
$$

where $p(x) \in L^{1}(-s, s) \cap C[-s, s]$. If the above equation has two points $s_{1}$ and $s_{2}$ in $[-s, s]$ satisfying $u\left(s_{1}\right)=0=u\left(s_{2}\right)$, then these points are said to be conjugate. It is wellknown that if there exists a pair of conjugate points in $[-s, s]$, then the classical Lyapunov inequality

$$
\int_{-s}^{s} p_{+}(x) d x>\frac{2}{s}
$$

holds, where $p_{+}(x):=\max (p(x), 0)$. Various extensions and improvements for the above result have been attempted; see, for example, Ha [1], Yang [2], and references there in. Among these extensions, Levin [3] and Das and Vatsala [4] extended the result for higher order equation

$$
(-1)^{M} u^{(2 M)}-p(x) u=0 \quad(-s \leq x \leq s) .
$$


For this case, we again call two distinct points $s_{1}$ and $s_{2}$ conjugate if there exists a nontrivial $C^{2 M}(-s, s) \cap C^{M-1}[-s, s]$ solution of (1.12) satisfying

$$
u^{(i)}\left(s_{1}\right)=0=u^{(i)}\left(s_{2}\right) \quad(i=0, \ldots, M-1) .
$$

We point out that the constant which appears in the generalized Lyapunov inequality by Levin [3] and Das and Vatsala [4] is the reverse of the Sobolev best embedding constant.

Corollary 1.4. If there exists a pair of conjugate points on $[-s, s]$ with respect to (1.12), then

$$
\int_{-s}^{s} p_{+}(x) d x>\frac{1}{C(M ;-s, s)}
$$

where $C(M ;-s, s)$ is the best constant of the Sobolev inequality (1.9).

Without introducing auxiliary equation $u^{(2 M)}+(-1)^{M-1} p_{+} u=0$ and the existence result of conjugate points as $[2,4]$, we can prove this corollary directly through the Sobolev inequality (the idea of the proof origins to Brown and Hinton [5, page 5]).

Proof of Corollary 1.4. Consider

$$
\begin{aligned}
\int_{s_{1}}^{s_{2}}\left(u^{(M)}(x)\right)^{2} d x & =\int_{s_{1}}^{s_{2}} p(x)(u(x))^{2} d x \leq\left(\sup _{s_{1} \leq x \leq s_{2}}|u(x)|\right)^{2} \int_{s_{1}}^{s_{2}} p_{+}(x) d x \\
& \leq C\left(M ; s_{1}, s_{2}\right) \int_{s_{1}}^{s_{2}}\left(u^{(M)}(x)\right)^{2} d x \int_{s_{1}}^{s_{2}} p_{+}(x) d x .
\end{aligned}
$$

In the second inequality, the equality holds for the function which attains the Sobolev best constant, so especially it is not a constant function. Thus, for this function, the first inequality is strict, and hence we obtain

$$
\frac{1}{C\left(M ; s_{1}, s_{2}\right)}<\int_{s_{1}}^{s_{2}} p_{+}(x) d x .
$$

Since

$$
\frac{1}{C(M ;-s, s)} \leq \frac{1}{C\left(M ; s_{1}, s_{2}\right)}<\int_{-s_{1}}^{s_{2}} p_{+}(x) d x \leq \int_{-s}^{s} p_{+}(x) d x
$$

we obtain the result.

Here, at the end of this section, we would like to mention some remarks about (1.12). The generalized Lyapunov inequality of the form (1.14) was firstly obtained by Levin [3] without proof; see Section 4 of Reid [6]. Later, Das and Vatsala [4] obtained the same inequality (1.14) by constructing Green's function for (BVP $(M))$. The expression of the Green's function of Proposition 1.1 is different from that of [4]. The expression of 
[4, Theorem 2.1] is given by some finite series of $x$ and $y$ on the other hand, the expression of Proposition 1.1 is by the determinant. This complements the results of [7-9], where the concrete expressions of Green's functions for the equation $(-1)^{M} u^{(2 M)}=f$ but different boundary conditions are given, and all of them are expressed by determinants of certain matrices as Proposition 1.1.

\section{Reproducing Kernel}

First we enumerate the properties of Green's function $G(x, y)$ of $(\operatorname{BVP}(M)) . G(x, y)$ has the following properties.

Lemma 2.1. Consider the following:

(1)

$$
\partial_{x}^{2 M} G(x, y)=0(-s<x, y<s, x \neq y)
$$

(2)

$$
\left.\partial_{x}^{i} G(x, y)\right|_{x= \pm s}=0(0 \leq i \leq M-1,-s<y<s)
$$

(3)

$$
\left.\partial_{x}^{i} G(x, y)\right|_{y=x-0}-\left.\partial_{x}^{i} G(x, y)\right|_{y=x+0}= \begin{cases}0 & (0 \leq i \leq 2 M-2), \\ (-1)^{M} & (i=2 M-1)(-s<x<s),\end{cases}
$$

(4)

$$
\left.\partial_{x}^{i} G(x, y)\right|_{x=y+0}-\left.\partial_{x}^{i} G(x, y)\right|_{x=y-0}= \begin{cases}0 & (0 \leq i \leq 2 M-2), \\ (-1)^{M} & (i=2 M-1)(-s<y<s) .\end{cases}
$$

Proof. For $k(1 \leq k \leq 2 M)$ and $-s<x, y<s, x \neq y$, we have from (1.5)

$$
\begin{aligned}
\partial_{x}^{k} G(x, y)=\frac{(-1)^{M}}{2}[ & (\operatorname{sgn}(x-y))^{k} K_{k}(|x-y|) \\
& \left.+D^{-1}\left\{\left|\begin{array}{c|c|c|}
K_{i+j}(2 s) & K_{i}(s-y) \\
\hline K_{k+j}(s+x) & 0
\end{array}\right|+\mid \begin{array}{c|c}
K_{i+j}(2 s) & K_{i}(s+y) \\
\hline(-1)^{k} K_{k+j}(s-x) & 0
\end{array}\right\}\right] .
\end{aligned}
$$


For $k=2 M$, noting the fact $K_{j}(x)=0 \quad(2 M \leq j)$, we have (1). Next, for $0 \leq k \leq M-1$ and $-s<y<s$, we have from $(2.5)$

$$
\begin{aligned}
& \left.\partial_{x}^{k} G(x, y)\right|_{x=-s} \\
& =\frac{(-1)^{M}}{2}\left[(-1)^{k} K_{k}(s+y)+D^{-1}\left\{\left|\begin{array}{c|c|}
K_{i+j}(2 s) & K_{i}(s-y) \\
\hline K_{k+j}(0) & 0
\end{array}\right|+\mid \begin{array}{c|c}
K_{i+j}(2 s) & K_{i}(s+y) \\
(-1)^{k} K_{k+j}(2 s) & 0
\end{array}\right\}\right] .
\end{aligned}
$$

Since $\left(K_{k}(0), \ldots, K_{k+M-1}(0)\right)=(0, \ldots, 0)$, we have

$$
\begin{aligned}
& \left.(-1)^{M+k} 2 \partial_{x}^{k} G(x, y)\right|_{x=-s}=K_{k}(s+y)+D^{-1}\left|\begin{array}{c|c}
K_{i+j}(2 s) & K_{i}(s+y) \\
\hline K_{k+j}(2 s) & 0
\end{array}\right|
\end{aligned}
$$

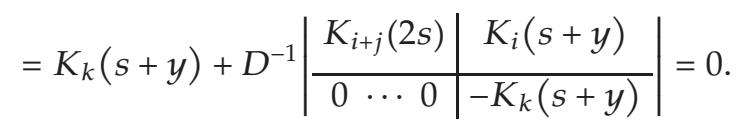

Note that subtracting the $k$ th row from $M$ th row, the second equality holds. Equation $\left.\partial_{x}^{k} G(x, y)\right|_{x=s}=0$ is shown by the same way. Hence, we have (2). For $0 \leq k \leq 2 M-1$, we have

$$
\begin{aligned}
& \left.\partial_{x}^{k} G(x, y)\right|_{y=x-0}-\left.\partial_{x}^{k} G(x, y)\right|_{y=x+0} \\
& \quad=\frac{(-1)^{M}}{2}\left(1-(-1)^{k}\right) K_{k}(0)= \begin{cases}0 & (0 \leq k \leq 2 M-2), \\
(-1)^{M} & (k=2 M-1)(-s<x<s),\end{cases}
\end{aligned}
$$

where we used the fact $K_{k}(0)=0(k \neq 2 M-1), 1(k=2 M-1)$. So we have (3), and (4) follows from (3).

Using Lemma 2.1, we prove that the functional space $H$ associated with inner norm $(\cdot, \cdot)_{M}$ is a reproducing kernel Hilbert space.

Lemma 2.2. For any $u \in H$, one has the reproducing property

$$
u(y)=(u(\cdot), G(\cdot, y))_{M}=\int_{-s}^{s} u^{(M)}(x) \partial_{x}^{M} G(x, y) d x \quad(-s \leq y \leq s) .
$$

Proof. For functions $u=u(x)$ and $v=v(x)=G(x, y)$ with $y$ arbitrarily fixed in $-s \leq y \leq s$, we have

$$
u^{(M)} v^{(M)}-u(-1)^{M} v^{(2 M)}=\left(\sum_{j=0}^{M-1}(-1)^{M-1-j} u^{(j)} v^{(2 M-1-j)}\right)^{\prime} .
$$


Integrating this with respect to $x$ on intervals $-s<x<y$ and $y<x<s$, we have

$$
\begin{aligned}
& \int_{-s}^{s} u^{(M)}(x) v^{(M)}(x) d x-\int_{-s}^{s} u(x)(-1)^{M} v^{(2 M)}(x) d x \\
&=\left[\sum_{j=0}^{M-1}(-1)^{M-1-j} u^{(j)}(x) v^{(2 M-1-j)}(x)\right]\left\{\mid \begin{array}{l}
x=y-0 \\
x=-s
\end{array}+\left\{\begin{array}{l}
x=s \\
x=y+0
\end{array}\right\}\right. \\
&= \sum_{j=0}^{M-1}(-1)^{M-1-j}\left[u^{(j)}(s) v^{(2 M-1-j)}(s)-u^{(j)}(-s) v^{(2 M-1-j)}(-s)\right] \\
&+\sum_{j=0}^{M-1}(-1)^{M-1-j} u^{(j)}(y)\left[v^{(2 M-1-j)}(y-0)-v^{(2 M-1-j)}(y+0)\right] .
\end{aligned}
$$

Using (1), (2), and (4) in Lemma 2.1, we have (2.9).

\section{Sobolev Inequality}

In this section, we give a proof of Theorem 1.2 and Corollary 1.3.

Proof of Theorem 1.2 and Corollary 1.3. Applying Schwarz inequality to (2.9), we have

$$
|u(y)|^{2} \leq \int_{-s}^{s}\left|\partial_{x}^{M} G(x, y)\right|^{2} d x \int_{-s}^{s}\left|u^{(M)}(x)\right|^{2} d x=G(y, y) \int_{-s}^{s}\left|u^{(M)}(x)\right|^{2} d x .
$$

Note that the last equality holds from (2.9); that is, substituting $(2.9), u(\cdot)=G(\cdot, y)$. Let us assume that

$$
C(M ;-s, s)=C(M)=\max _{|y| \leq s} G(y, y)=G(0,0)
$$

holds (this will be proved in the next section). From definition of $C(M)$, we have

$$
\left(\sup _{|y| \leq s}|u(y)|\right)^{2} \leq C(M) \int_{-s}^{s}\left|u^{(M)}(x)\right|^{2} d x
$$

Substituting $u(x)=G(x, 0) \in H$ in to the above inequality, we have

$$
\left(\sup _{|y| \leq s}|G(y, 0)|\right)^{2} \leq C(M) \int_{-s}^{s}\left|\partial_{x}^{M} G(x, 0)\right|^{2} d x=(C(M))^{2}
$$


Combining this and trivial inequality $(C(M))^{2}=(G(0,0))^{2} \leq\left(\sup _{|y| \leq s}|G(y, 0)|\right)^{2}$, we have

$$
(C(M))^{2} \leq\left(\sup _{|y| \leq s}|G(y, 0)|\right)^{2} \leq C(M) \int_{-s}^{s}\left|\partial_{x}^{M} G(x, 0)\right|^{2} d x=(C(M))^{2} .
$$

Hence, we have

$$
\left(\sup _{|y| \leq s}|G(y, 0)|\right)^{2}=C(M) \int_{-s}^{s}\left|\partial_{x}^{M} G(x, 0)\right|^{2} d x
$$

which completes the proof of Theorem 1.2 and Corollary 1.3.

Thus, all we have to do is to prove (3.2).

\section{Diagonal Value of Green's Function}

In this section, we consider the diagonal value of Green's function, that is, $G(x, x)$. From Proposition 1.1, we have for $M=1,2,3$

$$
G(1 ; x, x)=\frac{\left(s^{2}-x^{2}\right)}{2 s}, \quad G(2 ; x, x)=\frac{\left(s^{2}-x^{2}\right)^{3}}{24 s^{3}}, \quad G(2 ; x, x)=\frac{\left(s^{2}-x^{2}\right)^{5}}{650 s^{5}} .
$$

Thus, we can expect that $G(x, x)$ takes the form $G(M ; x, x)=$ const. $K_{0}(M ; 1+x) K_{0}(M ; 1-x)$. Precisely, we have the following proposition.

Proposition 4.1. Consider

$$
\begin{aligned}
G(x, x) & =(-1)^{M} D^{-1}\left|\begin{array}{c|c}
K_{i+j}(2 s) & K_{i}(s-x) \\
\hline K_{j}(s+x) & 0
\end{array}\right|=\left(\begin{array}{c}
2(M-1) \\
M-1
\end{array}\right) \frac{1}{K_{0}(2 s)} K_{0}(s+x) K_{0}(s-x) \\
& =\left(\begin{array}{c}
2(M-1) \\
M-1
\end{array}\right) \frac{1}{K_{0}(2 s)} \frac{\left(s^{2}-x^{2}\right)^{2 M-1}}{\{(2 M-1) !\}^{2}} .
\end{aligned}
$$

Hence,

$$
\begin{aligned}
C(M ;-s, s) & =\sup _{|x| \leq s} G(x, x)=G(0,0)=(-1)^{M} D^{-1}\left|\begin{array}{c|c}
K_{i+j}(2 s) & K_{i}(s) \\
\hline K_{j}(s) & 0
\end{array}\right| \\
& =\frac{s^{2 M-1}}{2^{2 M-1}(2 M-1) !}\left(\begin{array}{c}
2(M-1) \\
M-1
\end{array}\right)=\frac{s^{2 M-1}}{2^{2 M-1}(2 M-1)((M-1) !)^{2}}
\end{aligned}
$$

where $i, j$ satisfy $0 \leq i, j \leq M-1$. 
To prove this proposition, we prepare the following two lemmas.

Lemma 4.2. Let $u(x)=c_{1} G(x, x)$, where

$$
c_{1}^{-1}=(-1)^{M}\left(\begin{array}{c}
2(2 M-1) \\
2 M-1
\end{array}\right) D^{-1}\left|\begin{array}{ccc|c} 
& K_{i+j}(2 s) & 0 \\
& & & \vdots \\
& & & 0 \\
\hline 1 & 0 & \cdots & 0
\end{array}\right|,
$$

$(i, j$ satisfy $0 \leq i, j \leq M-1)$, then it holds that

$$
\begin{aligned}
& -u^{(2(2 M-1))}=1 \quad(-s<x<s) \\
& u^{(i)}( \pm s)=0 \quad(0 \leq i \leq 2 M-2) \\
& u^{(2 M-1)}(s)=-\left(\begin{array}{c}
2(M-1) \\
M-1
\end{array}\right) c_{1} .
\end{aligned}
$$

Lemma 4.3. Let $\tilde{u}(x)=c_{2} K_{0}(s+x) K_{0}(s-x)(-s<x<s)$, where $c_{2}^{-1}=\left(\begin{array}{c}2(2 M-1) \\ 2 M-1\end{array}\right)$, then it holds that (4.6) and $\tilde{u}^{(2 M-1)}(s)=-K_{0}(2 s) c_{2}$.

Proof of Proposition 4.1. From Lemmas 4.2 and 4.3, $u(x)=c_{1} G(x, x)$ and $\tilde{u}(x)=c_{2} K_{0}(s+$ $x) K_{0}(s-x)$ satisfy BVP $(2 M-1)$ (in case of $f(x)=1(-s<x<s)$ ). So we have

$$
\begin{gathered}
c_{1} G(x, x)=c_{2} K_{0}(s+x) K_{0}(s-x) \quad(-s<x<s), \\
\left(\begin{array}{c}
2(M-1) \\
M-1
\end{array}\right) c_{1}=K_{0}(2 s) c_{2} .
\end{gathered}
$$

Inserting (4.9) into (4.8), we have Proposition 4.1.

Proof of Lemma 4.2. Let

$$
u(x)=c_{1} G(x, x)=c_{1}(-1)^{M} D^{-1} v(x), \quad v(x)=\left|\begin{array}{c|c}
K_{i+j}(2 s) & K_{i}(s-x) \\
\hline K_{j}(s+x) & 0
\end{array}\right|
$$

then differentiating $v(x) k$ times we have

$$
v^{(k)}(x)=\sum_{l=0}^{k}(-1)^{l}\left(\begin{array}{l}
k \\
l
\end{array}\right) w_{k, l}(x), \quad w_{k, l}(x)=\mid \begin{array}{c|c}
K_{i+j}(2 s) & K_{l+i}(s-x) \\
\hline K_{k-l+j}(s+x) & 0
\end{array} .
$$


At first, for $k=2(2 M-1)$, we have

$$
\begin{aligned}
v^{(2(2 M-1))}(x)= & \sum_{l=0}^{2(2 M-1)}(-1)^{l}\left(\begin{array}{c}
2(2 M-1) \\
l
\end{array}\right) w_{2(2 M-1), l}(x) \\
= & \sum_{l=0}^{2 M-2}(-1)^{l}\left(\begin{array}{c}
2(2 M-1) \\
l
\end{array}\right) w_{2(2 M-1), l}(x)-\left(\begin{array}{c}
2(2 M-1) \\
2 M-1
\end{array}\right) w_{2(2 M-1), 2 M-1}(x) \\
& +\sum_{l=2 M}^{2(2 M-1)}(-1)^{l}\left(\begin{array}{c}
2(2 M-1) \\
l
\end{array}\right) w_{2(2 M-1), l}(x) .
\end{aligned}
$$

The first term vanishes because

$$
K_{2(2 M-1)-l+j}(s+x)=K_{2 M+(2 M-2-l+j)}(s+x)=0 \quad(0 \leq l \leq 2 M-2) .
$$

The third term also vanishes because

$$
K_{l+i}(s-x)=0 \quad(2 M \leq l \leq 2(2 M-1)) .
$$

Thus, we have

$$
\begin{aligned}
& v^{(2(2 M-1))}(x)=-\left(\begin{array}{c}
2(2 M-1) \\
2 M-1
\end{array}\right) w_{2(2 M-1), 2 M-1}(x),
\end{aligned}
$$

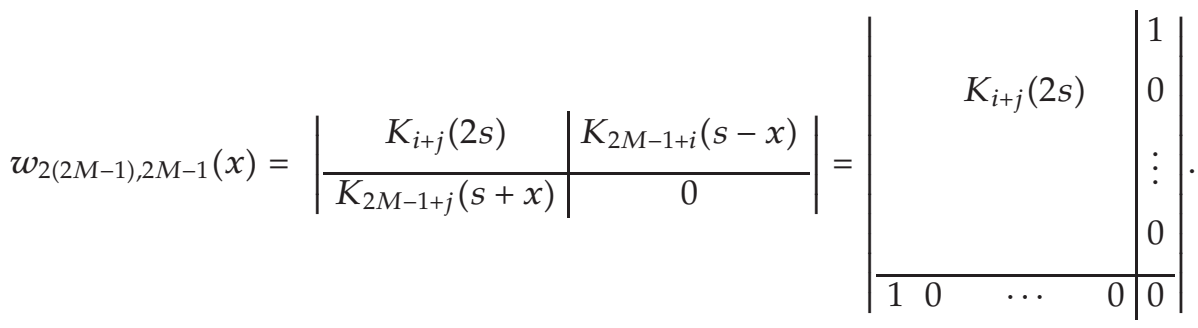

Hence, we have

$$
-u^{(2(2 M-1))}(x)=-c_{1}(-1)^{M} D^{-1} v^{(2(2 M-1))}(x)=1,
$$

by which we obtain (4.5). Next, for $0 \leq k \leq M-1$, we have

$$
v^{(k)}(s)=\sum_{l=0}^{k}(-1)^{l}\left(\begin{array}{l}
k \\
l
\end{array}\right) w_{k, l}(s), \quad w_{k, l}(s)=\left|\begin{array}{c|c}
K_{i+j}(2 s) & K_{l+i}(0) \\
\hline K_{k-l+j}(2 s) & 0
\end{array}\right| .
$$


Since $0 \leq l+i \leq 2 M-2$, we have $w_{k, l}(s)=0$. Thus, we have $v^{(k)}(s)=0(0 \leq k \leq M-1)$. For $M \leq k \leq 2 M-2$, we have

$$
v^{(k)}(s)=\sum_{l=0}^{M-1}(-1)^{l}\left(\begin{array}{l}
k \\
l
\end{array}\right) w_{k, l}(s)+\sum_{l=M}^{k}(-1)^{l}\left(\begin{array}{l}
k \\
l
\end{array}\right) w_{k, l}(s) .
$$

The first term vanishes because $K_{l+i}(0)=0(0 \leq l \leq M-1)$. Next, we show that the second term also vanishes. Let

$$
w_{k, l}(s)=\left|\begin{array}{c|c}
K_{j}(2 s) & 0 \\
\vdots & \vdots \\
K_{2 M-2-l+j}(2 s) & 0 \\
K_{2 M-1-l+j}(2 s) & 1 \\
K_{2 M-l+j}(2 s) & 0 \\
\vdots & \vdots \\
K_{M-1+j}(2 s) & 0 \\
\hline K_{k-l+j}(2 s) & 0
\end{array}\right| \quad(M \leq l \leq k \leq 2 M-2)
$$

Since $0 \leq k-l \leq 2 M-2-l$, two rows, including the last row, coincide, and hence we have $w_{k, l}(s)=0$. Thus, we have $v^{(k)}(s)=0(M \leq k \leq 2 M-2)$. So we have obtained $u^{(k)}(s)=0(0 \leq$ $k \leq 2 M-2)$. By the same argument, we have $u^{(k)}(-s)=0(0 \leq k \leq 2 M-2)$. Hence, we have (4.6). Finally, we will show (4.7). For $k=2 M-1$, noting $K_{l+i}(0)=0(0 \leq l \leq M-1)$, we have

$$
v^{(2 M-1)}(s)=\sum_{l=M}^{2 M-1}(-1)^{l}\left(\begin{array}{c}
2 M-1 \\
l
\end{array}\right) w_{2 M-1, l}(s),
$$

where

$$
\begin{aligned}
& w_{2 M-1, l}(s)=\left|\begin{array}{c|c}
K_{i+j}(2 s) & K_{l+i}(0) \\
\hline K_{2 M-1-l+j}(2 s) & 0
\end{array}\right|
\end{aligned}
$$

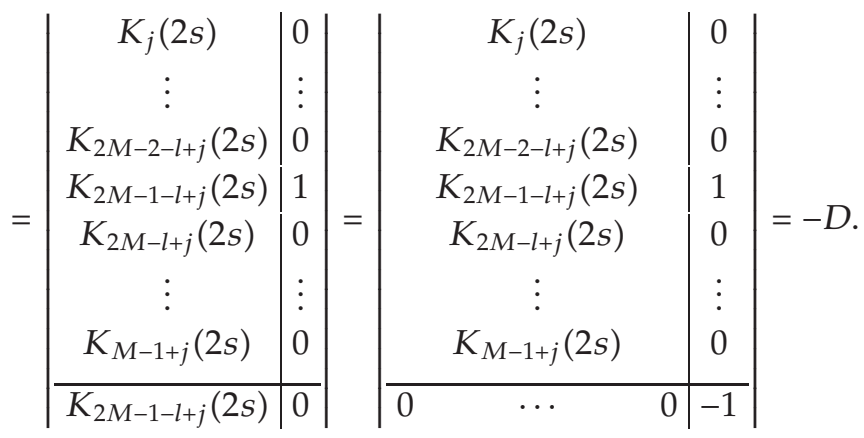


Thus, we obtain $w_{2 M-1, l}(s)=-D(M \leq l \leq 2 M-1)$. Hence we have

$$
\begin{aligned}
v^{(2 M-1)}(1) & =\sum_{l=M}^{2 M-1}(-1)^{l}\left(\begin{array}{c}
2 M-1 \\
l
\end{array}\right) w_{2 M-1, l}(s)=-D \sum_{l=M}^{2 M-1}(-1)^{l}\left(\begin{array}{c}
2 M-1 \\
l
\end{array}\right) \\
& =-D \sum_{l=M}^{2 M-2}(-1)^{l}\left\{\left(\begin{array}{c}
2 M-2 \\
l-1
\end{array}\right)+\left(\begin{array}{c}
2 M-2 \\
l
\end{array}\right)\right\}+D=(-1)^{M+1} D\left(\begin{array}{c}
2(M-1) \\
M-1
\end{array}\right),
\end{aligned}
$$

that is,

$$
u^{(2 M-1)}(s)=c_{1}(-1)^{M} D^{-1} v^{(2 M-1)}(s)=-\left(\begin{array}{c}
2(M-1) \\
M-1
\end{array}\right) c_{1} .
$$

This completes the proof of Lemma 4.2.

Proof of Lemma 4.3. Let

$$
u(x)=c_{2} K_{0}(s+x) K_{0}(s-x)=\frac{c_{2}}{((2 M-1) !)^{2}}\left(s^{2}-x^{2}\right)^{2 M-1} .
$$

Differentiating $u(x) k$ times, we have

$$
u^{(k)}(x)=c_{2} \sum_{l=0}^{k}(-1)^{l}\left(\begin{array}{l}
k \\
l
\end{array}\right) K_{k-l}(s+x) K_{l}(s-x)
$$

For $k=2(2 M-1)$, noting $K_{2(2 M-1)-l}(s+x)=0(0 \leq l \leq 2 M-2), K_{2 M-1}(s+x)=K_{2 M-1}(s-x)=1$, and $K_{l}(s-x)=0 \quad(2 M \leq l \leq 2(2 M-1))$, we have

$$
-u^{(2(2 M-1))}(x)=c_{2}\left(\begin{array}{c}
2(2 M-1) \\
2 M-1
\end{array}\right)=1 .
$$

Thus, we have (4.5). If $0 \leq k \leq 2 M-2$, then we have

$$
u^{(k)}(s)=c_{2} \sum_{l=0}^{k}(-1)^{l}\left(\begin{array}{l}
k \\
l
\end{array}\right) K_{k-l}(2 s) K_{l}(0)=0
$$

Since $u^{(k)}(-x)=(-1)^{k} u^{(k)}(x)$, we have $u^{(k)}(-s)=0(0 \leq k \leq 2 M-2)$. Hence, we have (4.6). If $k=2 M-1$, then we have

$$
u^{(2 M-1)}(s)=c_{2} \sum_{l=0}^{2 M-1}(-1)^{l}\left(\begin{array}{c}
2 M-1 \\
l
\end{array}\right) K_{2 M-1-l}(2 s) K_{l}(0)=-c_{2} K_{0}(2 s) .
$$

This proves Lemma 4.3 . 


\section{Appendices}

\section{A. Deduction of $(1.5)$}

In this section, (1.5) in Proposition 1.1 is deduced. Suppose that $(\operatorname{BVP}(M))$ has a classical solution $u(x)$. Introducing the following notations:

$$
\begin{aligned}
& \mathbf{u}={ }^{t}\left(u_{0}, \ldots, u_{2 M-1}\right), \quad u_{i}=u^{(i)} \quad(0 \leq i \leq 2 M-1), \\
& \mathbf{e}={ }^{t}(0, \ldots, 0,1) \quad(2 M \times 1 \text { matrix }), \\
& \mathbf{N}=\left(\begin{array}{cccc}
0 & 1 & & \\
& 0 & \ddots & \\
& & \ddots & 1 \\
& & & 0
\end{array}\right) \quad(2 M \times 2 M \text { nilpotent matrix }),
\end{aligned}
$$

$(\operatorname{BVP}(M))$ is rewritten as

$$
\begin{gathered}
\mathbf{u}^{\prime}=\mathbf{N u}+\mathbf{e}(-1)^{M} f(x) \quad(-s<x<s), \\
u_{i}( \pm s)=0 \quad(0 \leq i \leq M-1) .
\end{gathered}
$$

Let the fundamental solution $\mathbf{E}(x)$ be expressed as $\mathbf{E}(x)=\exp (\mathbf{N} x)=\mathbf{K}(x) \mathbf{K}(0)^{-1}$, where

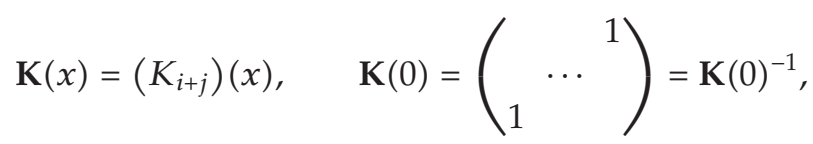

then $i, j$ satisfy $0 \leq i, j \leq 2 M-1$. $\mathbf{E}(x)$ satisfies the initial value problem $\mathbf{E}^{\prime}=\mathbf{N E}, \mathbf{E}(0)=\mathbf{I}$. $\mathbf{I}$ is a unit matrix. Solving (A.2), we have

$$
\begin{aligned}
& \mathbf{u}(x)=\mathbf{E}(x+s) \mathbf{u}(-s)+\int_{-s}^{x} \mathbf{E}(x-y) \mathbf{e}(-1)^{M} f(y) d y, \\
& \mathbf{u}(x)=\mathbf{E}(x-s) \mathbf{u}(s)-\int_{x}^{s} \mathbf{E}(x-y) \mathbf{e}(-1)^{M} f(y) d y,
\end{aligned}
$$

or equivalently, for $0 \leq i \leq 2 M-1$, we have

$$
\begin{aligned}
& u_{i}(x)=\sum_{j=0}^{2 M-1} K_{i+j}(x+s) u_{2 M-1-j}(-s)+\int_{-s}^{x}(-1)^{M} K_{i}(x-y) f(y) d y, \\
& u_{i}(x)=\sum_{j=0}^{2 M-1} K_{i+j}(x-s) u_{2 M-1-j}(s)-\int_{x}^{s}(-1)^{M} K_{i}(x-y) f(y) d y .
\end{aligned}
$$


Employing the boundary conditions (A.2), we have

$$
\begin{aligned}
& u_{i}(x)=\sum_{j=0}^{M-1} K_{i+j}(x+s) u_{2 M-1-j}(-s)+\int_{-s}^{x}(-1)^{M} K_{i}(x-y) f(y) d y, \\
& u_{i}(x)=\sum_{j=0}^{M-1} K_{i+j}(x-s) u_{2 M-1-j}(s)-\int_{x}^{s}(-1)^{M} K_{i}(x-y) f(y) d y .
\end{aligned}
$$

In particular, if $i=0$, then we have

$$
\begin{aligned}
& u_{0}(x)=\sum_{j=0}^{M-1} K_{j}(x+s) u_{2 M-1-j}(-s)+\int_{-s}^{x}(-1)^{M} K_{0}(x-y) f(y) d y, \\
& u_{0}(x)=\sum_{j=0}^{M-1} K_{j}(x-s) u_{2 M-1-j}(s)-\int_{x}^{s}(-1)^{M} K_{0}(x-y) f(y) d y .
\end{aligned}
$$

On the other hand, using the boundary conditions (A.2) again, we have

$$
\begin{aligned}
& 0=u_{i}(s)=\sum_{j=0}^{M-1} K_{i+j}(2 s) u_{2 M-1-j}(-s)+\int_{-s}^{s}(-1)^{M} K_{i}(s-y) f(y) d y, \\
& 0=u_{i}(-s)=\sum_{j=0}^{M-1} K_{i+j}(-2 s) u_{2 M-1-j}(s)-\int_{-s}^{s}(-1)^{M} K_{i}(-s-y) f(y) d y .
\end{aligned}
$$

Solving the above linear system of equations with respect to $u_{2 M-1-i}(-s)$, $u_{2 M-1-i}(s)(0 \leq i \leq M-1)$, we have

$$
\begin{aligned}
& \left(u_{2 M-1-i}\right)(-s)=-\int_{-s}^{s}(-1)^{M}\left(K_{i+j}\right)^{-1}(2 s)\left(K_{i}\right)(s-y) f(y) d y, \\
& \left(u_{2 M-1-i}\right)(s)=\int_{-s}^{s}(-1)^{M}\left(K_{i+j}\right)^{-1}(-2 s)\left(K_{i}\right)(-s-y) f(y) d y .
\end{aligned}
$$

Substituting (A.9) into (A.7), we have

$$
\begin{aligned}
u_{0}(x)= & -\int_{-s}^{s}(-1)^{M}\left(K_{j}\right)(x+s)\left(K_{i+j}\right)^{-1}(2 s)\left(K_{i}\right)(s-y) f(y) d y \\
& +\int_{-s}^{x}(-1)^{M} K_{0}(|x-y|) f(y) d y, \\
u_{0}(x)= & \int_{-s}^{s}(-1)^{M}\left(K_{j}\right)(x-s)\left(K_{i+j}\right)^{-1}(-2 s)\left(K_{i}\right)(-s-y) f(y) d y \\
& +\int_{x}^{s}(-1)^{M} K_{0}(|x-y|) f(y) d y .
\end{aligned}
$$


Taking an average of the above two expressions and noting $u(x)=u_{0}(x)$, we obtain (1.4), where Green's function $G(x, y)$ is given by

$$
\begin{gathered}
G(x, y)=\frac{(-1)^{M}}{2}\left[K_{0}(|x-y|)-\left(K_{j}\right)(x+s)\left(K_{i+j}\right)^{-1}(2 s)\left(K_{i}\right)(s-y)\right. \\
\left.+\left(K_{j}\right)(x-s)\left(K_{i+j}\right)^{-1}(-2 s)\left(K_{i}\right)(-s-y)\right] .
\end{gathered}
$$

Using properties $K_{i}(-x)=(-1)^{i+1} K_{i}(x)$, we have

$$
\begin{gathered}
\left(K_{j}\right)(x-s)=-\left(K_{j}\right)(s-x)\left((-1)^{i} \delta_{i j}\right), \\
\left(K_{i+j}\right)(-2 s)=\left((-1)^{i+j+1} K_{i+j}\right)(2 s)=-\left((-1)^{i} \delta_{i j}\right)\left(K_{i+j}\right)(2 s)\left((-1)^{i} \delta_{i j}\right), \\
\left(K_{i}\right)(-s-y)=\left((-1)^{i+1} K_{i}\right)(s+y)=-\left((-1)^{i} \delta_{i j}\right)\left(K_{i}\right)(s+y),
\end{gathered}
$$

where $\delta_{i j}$ is Kronecker's delta defined by $\delta_{i j}=1(i=j), 0(i \neq j)$. Inserting these three relations into (A.11), we have

$$
\begin{gathered}
G(x, y)=\frac{(-1)^{M}}{2}\left[K_{0}(|x-y|)-\left(K_{j}\right)(s+x)\left(K_{i+j}\right)^{-1}(2 s)\left(K_{i}\right)(s-y)\right. \\
\left.-\left(K_{j}\right)(s-x)\left(K_{i+j}\right)^{-1}(2 s)\left(K_{i}\right)(s+y)\right] .
\end{gathered}
$$

Applying the relation

$$
{ }^{t} \mathbf{a} \mathbf{A}^{-1} \mathbf{b}=-\frac{\left|\begin{array}{c|c}
\mathbf{A} & \mathbf{b} \\
{ }^{t} \mathbf{a} & 0
\end{array}\right|}{|\mathbf{A}|}
$$

where $\mathbf{A}$ is any $N \times N$ regular matrix and $\mathbf{a}$ and $\mathbf{b}$ are any $N \times 1$ matrices, we have (1.5).

\section{B. Deduction of (1.6)}

To prove (1.6), we show

$$
K_{0}(x-y)=-D^{-1}\left\{\left|\begin{array}{c|c|}
K_{i+j}(2 s) & K_{i}(s-y) \\
\hline K_{j}(s+x) & 0
\end{array}\right|-\left|\begin{array}{c|c}
K_{i+j}(2 s) & K_{i}(s+y) \\
\hline K_{j}(s-x) & 0
\end{array}\right|\right\} \quad(-s<x, y<s) .
$$


Let $x \geq y$. If (B.1) holds, substituting it to (1.5), replacing $x$ with $x \vee y, y$ with $x \wedge y$, then we obtain (1.6). The case $x \leq y$ is shown in a similar way. Let $y(-s \leq y \leq s)$ be fixed, and let $u(x)=K_{0}(x-y)$. Then $u$ satisfies

$$
\begin{gathered}
u^{(2 M)}=0 \quad(-s<x<s), \\
u^{(i)}(-s)=(-1)^{i+1} K_{i}(s+y), \quad u^{(i)}(s)=K_{i}(s-y) \quad(0 \leq i \leq M-1) .
\end{gathered}
$$

On the other hand, let

$$
v(x)=-D^{-1}\left\{\left|\begin{array}{c|c}
K_{i+j}(2 s) & K_{i}(s-y) \\
\hline K_{j}(s+x) & 0
\end{array}\right|-\left|\begin{array}{c|c}
K_{i+j}(2 s) & K_{i}(s+y) \\
\hline K_{j}(s-x) & 0
\end{array}\right|\right\} .
$$

Differentiating $v k$ times with respect to $x$, we have

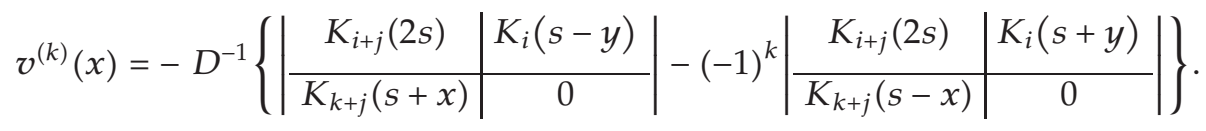

For $k=2 M$, noticing $K_{k+j}(s+x)=K_{k+j}(s-x)=0$, we have $v^{(2 M)}(x)=0$. For $0 \leq k \leq M-1$, we have

$$
\begin{aligned}
& v^{(k)}(-s)=-D^{-1}\left\{\left|\begin{array}{c|c|}
K_{i+j}(2 s) & K_{i}(s-y) \\
\hline K_{k+j}(0) & 0
\end{array}\right|-(-1)^{k}\left|\begin{array}{c|c}
K_{i+j}(2 s) & K_{i}(s+y) \\
\hline K_{k+j}(2 s) & 0
\end{array}\right|\right\}
\end{aligned}
$$

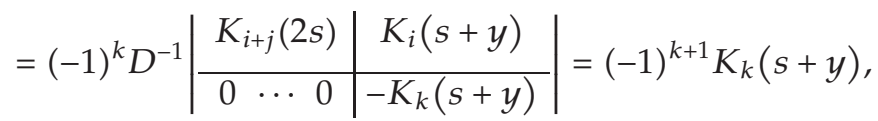

where we used $K_{k+j}(0)=0$. Similarly, for $0 \leq k \leq M-1$, we have $v^{(k)}(s)=K_{k}(s-y)$. So $v(x)$ satisfies

$$
\begin{gathered}
v^{(2 M)}=0 \quad(-s<x<s), \\
v^{(i)}(-s)=(-1)^{i+1} K_{i}(s+y), \quad v^{(i)}(s)=K_{i}(s-y) \quad(0 \leq i \leq M-1) .
\end{gathered}
$$

which is the same equation as (B.2). Hence, we have $v(x)=u(x)$.

\section{References}

[1] C.-W. Ha, "Eigenvalues of a Sturm-Liouville problem and inequalities of Lyapunov type," Proceedings of the American Mathematical Society, vol. 126, no. 12, pp. 3507-3511, 1998.

[2] X. Yang, "On inequalities of Lyapunov type," Applied Mathematics and Computation, vol. 134, no. 2-3, pp. 293-300, 2003.

[3] A. J. Levin, "Distribution of the zeros of solutions of a linear differential equation," Soviet Mathematics, vol. 5, pp. 818-821, 1964. 
[4] K. M. Das and A. S. Vatsala, "Green's function for $n-n$ boundary value problem and an analogue of Hartman's result," Journal of Mathematical Analysis and Applications, vol. 51, no. 3, pp. 670-677, 1975.

[5] R. C. Brown and D. B. Hinton, "Lyapunov inequalities and their applications," in Survey on Classical Inequalities, T. M. Rassias, Ed., vol. 517 of Math. Appl., pp. 1-25, Kluwer Academic Publishers, Dordrecht, The Netherlands, 2000.

[6] W. T. Reid, "A generalized Liapunov inequality," Journal of Differential Equations, vol. 13, pp. 182-196, 1973.

[7] Y. Kametaka, H. Yamagishi, K. Watanabe, A. Nagai, and K. Takemura, "Riemann zeta function, Bernoulli polynomials and the best constant of Sobolev inequality," Scientiae Mathematicae Japonicae, vol. 65, no. 3, pp. 333-359, 2007.

[8] A. Nagai, K. Takemura, Y. Kametaka, K. Watanabe, and H. Yamagishi, "Green function for boundary value problem of $2 M$-th order linear ordinary differential equations with free boundary condition," Far East Journal of Applied Mathematics, vol. 26, no. 3, pp. 393-406, 2007.

[9] Y. Kametaka, K. Watanabe, A. Nagai, and S. Pyatkov, "The best constant of Sobolev inequality in an $n$ dimensional Euclidean space," Scientiae Mathematicae Japonicae, vol. 61, no. 1, pp. 15-23, 2005. 